\title{
Convergencia económica en América Central: Evidencia empírica, 1975-2005
}

\author{
Mirta Macías Ruano*, Luis Alonso Reyes**
}

\section{RESUMEN}

Se presentan hechos estilizados del crecimiento económico y la convergencia interregional para Centroamérica expuestos en la literatura empírica de crecimiento. Para ello, se hace un análisis de corte transversal para el período de referencia 1975-2005, y con ello establecer la existencia o no de convergencia en los niveles del Producto Interno Bruto (PIB) per cápita entre los países analizados. Adicionalmente, se indaga sobre el efecto de algunos determinantes sobre el crecimiento económico durante el período 1993-2005.

Los resultados indican que en el largo plazo (1975-2005) se acepta la hipótesis de convergencia, pero este proceso se revierte a partir de la década de 1980 y los valores de los coeficientes se reducen drásticamente en el último período 19952005. En cuanto a las determinantes del crecimiento económico se encontró que las variables educativas, el gasto en formación bruta de capital fijo y la tasa crecimiento poblacional son significativas estadísticamente.

Palabras Clave: Crecimiento económico, convergencia, desigualdades, divergencia

\section{ABSTRACT}

In this paper we present stylized facts of economic growth and interregional convergence for Central America exposed in the empirical literature growth. This makes a cross-sectional analysis for the reference period 1975-2005 and thus try to establish whether or not there is convergence between countries. Additionally, it includes an analysis with variable conditions to capture any differences between the stationary states of the various Central American economies during the period 19932005.

"Universidad Nacional Autónoma de Honduras-Valle de Sula. Facultad de Ciencias Económicas. Escuela de Economía. mirtamaru@hotmail.com

Universidad Nacional Autónoma de Honduras. Dirección de Investigación Científica.

Ireyes64@hotmail.com 
The results indicate that the long-term (1975-2005) one accepts the hypothesis of convergence, but this process is reversed from the 1980's and the speed of convergence is reduced in the last period, 1995-2005. As for the determinants of economic growth, we found that educational variables, spending on gross fixed capital formation and population growth rate are statistically significant.

Key Words: Economic growth, inequality, convergence, divergence 


\section{INTRODUCCIÓN}

Tras la década de 1980, América Central en los últimos años ha logrado recuperar su crecimiento económico, pero éste ha sido moderado e insuficiente. A excepción de Costa Rica, en el resto de países persisten antiguos problemas como la falta de competitividad y la inequidad en la distribución de la renta. En los últimos años el tema de la convergencia económica ha suscitado un creciente debate en un contexto donde las disparidades entre países crecen aún más. Al respecto, las rezones son variadas, no obstante, la más aceptada y que ha sido desarrollada en trabajos como el de Barro y Sala-i-Martín es la que se refiere a los factores que explican el crecimiento económico.

Bajo estas consideraciones, una de las cuestiones más debatidas es sí las disparidades entre países o regiones tienden a reducirse en el mediano y largo plazo sin o con la intervención de la acción pública. En tal sentido, nuestro interés por el tema del crecimiento económico se centra en primer lugar, en mostrar algunos hechos estilizados en cuanto a la economía centroamericana en términos de convergencia y/o divergencia en el período analizado.

Para ello se realiza un análisis de corte transversal para el período de referencia 1975-2005, y con ello intentar establecer la existencia o no de convergencia en los niveles del Producto Interno Bruto (PIB) per cápita en la región. En segundo lugar se pretende contribuir al debate sobre el tema de convergencia, un tópico poco abordado en Centroamérica, por lo que es de particular relevancia en el caso de que exista poca evidencia de convergencia, además de explicar algunas razones del por qué algunos países muestran un mayor dinamismo mientras que otros se quedan rezagados.

El trabajo se ha organizado en tres apartados: en el primero se presentan los fundamentos teóricos en los que se basa el estudio; en el segundo se analiza la evidencia empírica del crecimiento económico regional centroamericano, y adicionalmente se indaga sobre el efecto que algunos determinantes tienen sobre el crecimiento económico durante el período 1993-2005. Finalmente se presentan las conclusiones de los resultados obtenidos.

\section{FUNDAMENTO TEÓRICO}

Estudios recientes sobre el crecimiento económico buscan encontrar explicaciones al comportamiento de esta variable en el tiempo y que determinantes influyen más en ella. Estos estudios han basado sus trabajos en la modelización del crecimiento, 
entre ellos los modelos neoclásicos son un referente en este tema. Uno de los supuestos básicos de los modelos neoclásicos de crecimiento es que el producto 0 renta per cápita de las distintas economías convergen en el largo plazo. Dichos modelos predicen que los países pobres crecerán más deprisa que los ricos sólo bajo determinadas condiciones. En 1956, Solow y Swan propusieron iniciar el estudio del crecimiento económico asumiendo una función de producción neoclásica estándar con retornos constantes a escala y rendimientos marginales decrecientes al capital. Tomando las tasas de ahorro y de crecimiento de la población como variables exógenas, demostraron que estas dos variables determinan el nivel del estado estacionario del producto o renta per cápita.

Debido a que el ahorro y las tasas de crecimiento de la población varían entre los países, de igual forma alcanzan diferentes estados estacionarios. Este modelo busca encontrar las variables relevantes que inciden en el crecimiento económico de un país o región, algunas ayudan a mejorar la situación sólo en el corto plazo y otras afectan las tasas de crecimiento en el largo plazo. Se incluyen las variables que el modelo considera significativas, mostrando la incidencia de éstas en el proceso de crecimiento.

El modelo utiliza una función de producción Cobb-Douglas: $Y=A K^{a} L^{1-a}$ donde $\mathrm{K}$ es capital, L la fuerza de trabajo y A es el nivel tecnológico ${ }^{1}$. La función opera bajo los supuestos de retornos constantes a escala y rendimientos marginales decrecientes al capital y al trabajo. Solow plantea una ecuación relevante dentro de su modelo: $\Delta K=s Y-\delta K$, donde $\mathrm{K}$ es el capital, Yes el producto o ingreso de la economía de un país o región, $s Y$ representa la inversión efectiva que es igual al ingreso multiplicado por la tasa de ahorro y $\delta$ es la tasa de depreciación. Esta ecuación refleja la acumulación de capital en términos absolutos.

Se toma que $k=(K / A L)$ y teniendo en cuenta que $\Delta L / L=n$ y $\Delta A / A=g$, derivando se obtiene: $\quad \Delta K=s y-(\delta+n+g) k \quad$ donde $(\delta+n+g) k$ muestra la cantidad de inversión necesaria para mantener el capital constante. Aumentos de depreciación, tendrían efectos de disminución de la acumulación de capital, y por lo tanto, un menor estado estacionario del capital. Aumentos en la tasa de crecimiento de la población, causarían una disminución de la acumulación de capital per cápita efectivo.

Es necesario, que la inversión efectiva pueda sostener los movimientos o la

\footnotetext{
${ }^{1}$ La función opera bajo los supuestos de retornos constantes a escala y rendimientos marginales decrecientes al capital y al trabajo. Además cumple con la condición de Inada, es decir, el producto marginal del capital es 0 cuando Kes alto, por otra parte, cuando K es demasiado bajo, el producto marginal es muy alto.
} 
depreciación misma, así como el crecimiento de la población y la nueva tecnología que necesitan inversión física para producirla. Si tenemos altas tasas de crecimiento de la población, es difícil que el capital per cápita efectivo crezca, ya que habrá mayor maquinaria que repartir entre los nuevos individuos potencialmente productivos que entran al mercado.

A partir de la ecuación anterior se basa en lo que suele llamarse el equilibrio del estado estacionario. Ésto es la condición del modelo en que finaliza el aumento del capital reflejado en la ecuación de acumulación de capital per cápita, que termina con un capital fijo sin variaciones adicionales $(\Delta K=0 \quad s y=(\delta+n+g) k)$.

El equilibrio en el modelo de Solow es la senda de la convergencia de los países: una economía, mediante la propiedad de productos marginales decrecientes, tiende a decrecer su producción marginal, o dicho en otros términos, la producción total crece cada vez menos. Por lo que sy, tiende también a crecer menos, lo que eventualmente hace que se iguale a $(\delta+n+g) k$. Esta condición, mantiene el stock de capital per cápita efectivo constante, sin variaciones.

Sin embargo, en estado estacionario, es posible afirmar que el producto per cápita crece a la tasa de crecimiento de la tecnología, y el producto total crece a la tasa de crecimiento de la población y de la tecnología. El aporte de estas variables exógenas logra explicar el crecimiento en el largo plazo, es decir, cuando la economía alcanza su capital estacionario.

Basados en el modelo Solow-Swan, Barro y Sala-i-Martín (1995) desarrollan los conceptos de convergencia conocidos como convergencia Beta $(\beta)$ y convergencia Sigma $(\sigma)$. Se dice que existe convergencia sigma cuando la dispersión del producto o renta real per cápita de un grupo de países o regiones tiende a disminuir a lo largo del tiempo, midiéndose normalmente mediante la desviación estándar; y convergencia beta, si para ese grupo de países existe una relación inversa entre la tasa de crecimiento del PIB o renta real per cápita y el nivel inicial de ésta, o, en otras palabras, cuando los países pobres tienden a crecer más rápidamente que los países ricos, de forma que todos los países tienden a un mismo nivel, que suele llamarse estado estacionario.

A pesar de ser diferentes, los conceptos de beta y sigma convergencia están relacionados. Asumiendo que la tasa de crecimiento del producto o renta per cápita de un país o una región en un periodo dado se escriba como:

$$
\log \left(y_{i, t}\right)-\log \left(y_{i, 0}\right)=\alpha-\beta \log \left(y_{i, 0}\right)+\mu_{i, t}
$$


La tasa de crecimiento estará dada por la diferencia de los logaritmos del producto 0 renta per cápita final menos el valor inicial. Para aceptar la hipótesis de Beta convergencia deberá existir una relación inversa entre la tasa de crecimiento del producto o renta per cápita y su valor inicial. Cuanto mayor sea $\beta$, mayor será la tendencia hacia la convergencia $(\beta>0)$, pero el supuesto es que $\beta$ está entre 0 y $1^{2}$.

La convergencia beta es una condición necesaria para la existencia de convergencia sigma, pero no suficiente. El anterior concepto de convergencia beta se refiere a la denominada convergencia beta absoluta o no condicional. Pero es posible distinguir también el concepto de convergencia beta condicional, que se produce cuando tan sólo existe una correlación parcial negativa entre la tasa de crecimiento del PIB o renta per cápita de un país y su nivel inicial.

Es decir, si al realizar una regresión entre la tasa de crecimiento del producto o renta per cápita, su nivel inicial, y cierto número de variables explicativas, encontramos que el coeficiente del valor inicial es negativo, se puede decir que las economías en conjunto muestran convergencia condicional.

El modelo propuesto por estos autores para el análisis de la convergencia es una ecuación no lineal de la siguiente forma:

$$
\left(\frac{\ln y_{i, t}-\ln y_{i, 0}}{T}\right)=\alpha-\left(\frac{1-e^{-\beta \cdot T}}{T}\right) \ln y_{i, 0}+\mu_{i, T}
$$

donde $y_{i, t}$ es la renta o producto per cápita del país o región i en el momento $t$, T es el intervalo de tiempo entre el momento inicial 0 y $t$ y $\mu_{i, T}$ representa el promedio de los términos de error durante el intervalo $T$.

Según Sala-i-Martín (2000), la razón de utilizar esta ecuación es que el parámetro $\beta$ da directamente la velocidad de convergencia de la economía sin importar la duración del periodo de estimación y, además, que el coeficiente del logaritmo del valor inicial $\left(1-\mathrm{e}^{-\beta T}\right) / T$, es una función decreciente de la duración del periodo de estimación, además que esta es precisamente la ecuación que predice el modelo neoclásico.

\footnotetext{
${ }^{2}$ Sala-i-Martín (2000) plantea que cuando $\beta<1$ se elimina la posibilidad de "adelantamientos sistemáticos". Es decir, que los países que comienzan siendo pobres acaben siendo más ricos que los que comenzaron siendo ricos. Algunos economistas sostienen que en largo plazo este hecho puede suceder, pero debido a múltiples factores y no sólo al hecho de que por ser pobre, en el futuro se volverá rico, nadie piensa que habrá adelantamientos sistemáticos.
} 


\section{EVIDENCIAEMPÍRICA}

Los datos del PIB per cápita están expresados en valores de PPA(poder de paridad adquisitiva) en dólares a precios del 2000. Los datos ajustados del PIB per cápita de cada país permiten comparar niveles de actividad real entre los países analizados ${ }^{3}$. América Central es una región de contrastes, las disparidades entre los niveles de crecimiento económico y de bienestar en general están determinadas por diversos factores que influyen o influyeron particularmente sobre cada uno de los países, entre ellos, las guerras en la década de los ochenta, los desastres naturales en los años noventa, así como los procesos de reestructuración económica.

Gráfico Nº1. Tendencia del PIB per cápita entre 1975-2005. (PPA, en dólares a precios constantes de 2000)

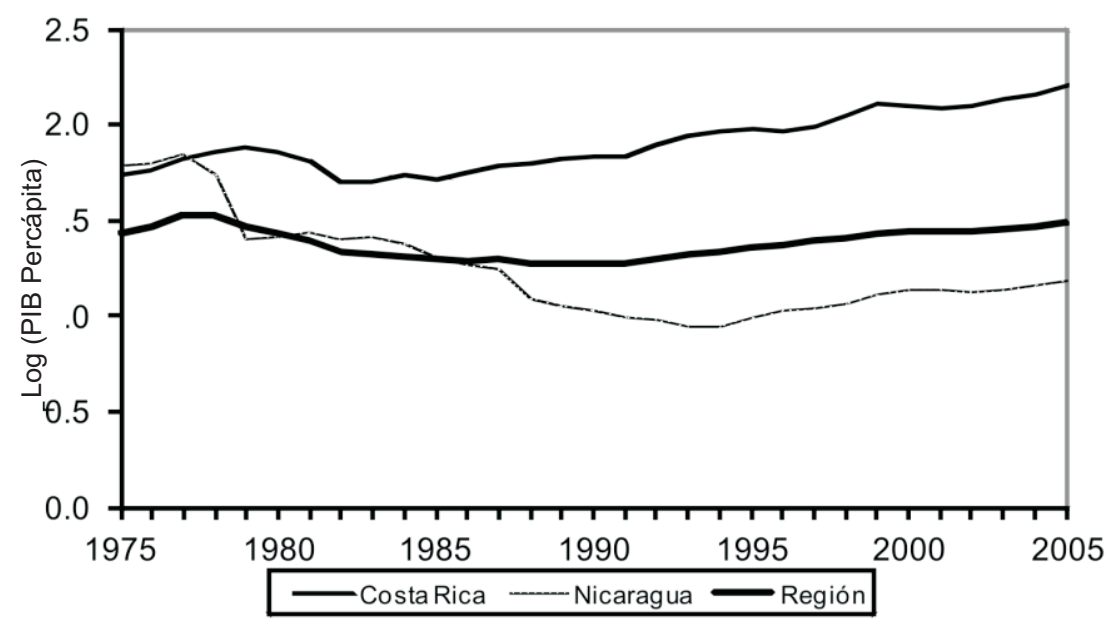

Fuente: Elaboración propia, WDI 2006.

En el gráfico $\mathrm{N}^{\circ} 1$ se observa la evolución del PIB per cápita en la región. Al contrastar los datos vemos que Costa Rica mantiene una tendencia de crecimiento, mientras tanto, en Nicaragua ha decrecido, incluso por debajo del promedio regional. Los patrones seguidos por los países indican una evolución heterogénea del producto per cápita. Nicaragua tiene un claro descenso en su nivel a partir de finales de la década de 1970 lo que concuerda con su situación socio-política de esa época. Por su parte, la tendencia regional parece más estable durante el periodo analizado, pero al igual que Nicaragua sufre una contracción durante la

\footnotetext{
${ }^{3}$ La información procede de la base de datos de Banco Mundial (Indicadores de Desarrollo Mundial, 2006) y Anuario Estadístico de la CEPAL (2006)
} 
primera mitad de los años ochenta, volviendo a crecer a partir de la década de 1990 en adelante. Romer (2006) argumenta que Latinoamérica exhibe una de las pautas de crecimiento más difíciles de describir. Muchos países del área durante la década de los sesenta y setenta tuvieron elevadas tasas de crecimiento para luego caer en la década siguiente y volver a crecer en los años noventa, no sin mostrar en periodos cortos fuertes contracciones. El gráfico $\mathrm{N}^{\circ} 2$ muestra los componentes cíclicos del producto per cápita para Costa Rica, Nicaragua y el promedio regional y se reflejan las épocas de expansión y recesión sufridas durante el período analizado ${ }^{4}$.

De los resultados se puede deducir que, en general, los periodos de recesión y expansión coinciden entre países. Sin embargo, Costa Rica parece adelantada, lo que es congruente, sí se toma en cuenta que es el país con el mayor producto per cápita de la región. Por otro lado, Nicaragua tuvo una recesión muy larga en los 80 , de la que empieza a recuperarse al final de la muestra analizada. También se observa una significativa reducción de la volatilidad, lo que indica que los ciclos económicos son menos pronunciados al final del período.

Gráfico $\mathbf{N}^{\circ}$ 2. Componentes cíclicos del PIB per cápita en Centroamérica, 1975-2005

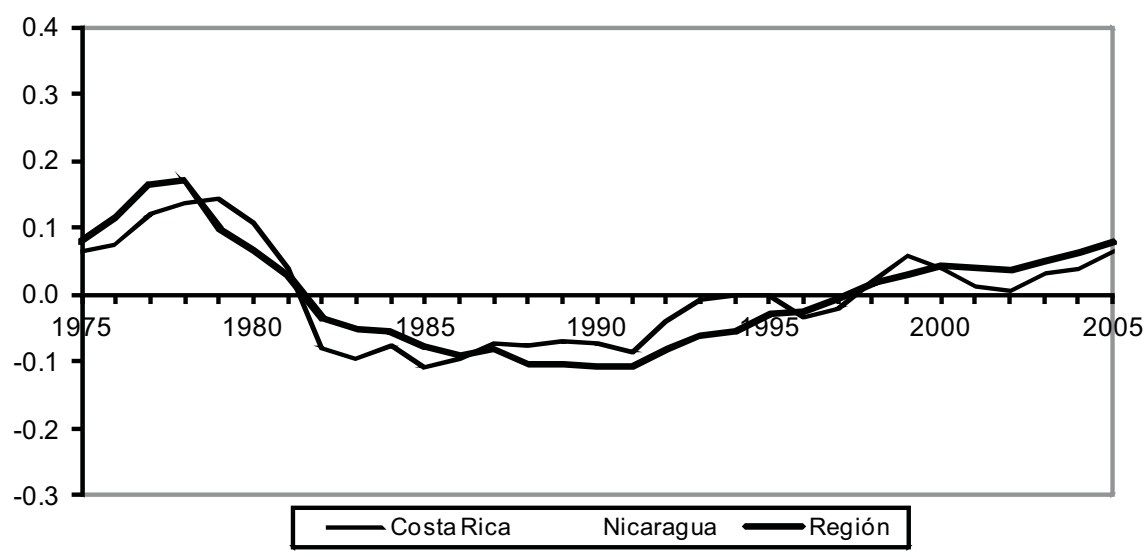

Fuente: Elaboración propia, WDI 2006.

En el informe del crecimiento mundial correspondiente a 1997 del Banco Mundial, Nicaragua es catalogada como un desastre económico, el crecimiento promedio

\footnotetext{
${ }^{4}$ Una forma de calcular el componente cíclico del PIB per cápita es estimar por mínimos cuadrados ordinarios una ecuación lineal de la forma: $Y_{t}=\alpha+g_{t}+\mu_{t}$ donde $Y_{t}$ representa el logaritmo del PIB per cápita en el periodo $t, t$ es el año en que se está, a y g son los parámetros a estimar y $\mu_{t}$ son los residuos de la regresión que se interpretan como el componente cíclico del producto per cápita. Despejando se obtiene que $\mu_{t}=Y_{t}-\left(a+g_{t}\right)$, es decir, el valor mostrado menos la tendencia del PIB per cápita durante el período t.
} 
entre 1960 y 1996 fue de -1,29 y la relación entre el valor final e inicial fue de 0,62 valores aproximados a los encontrados en este trabajo.

Cuadro N¹. Patrón Regional del crecimiento: 1975-2005

\begin{tabular}{|c|c|c|}
\hline País & Crecimiento $\mathbf{1 9 7 5}$ & Niños controles \\
\hline Costa Rica & 1.55 & 1.59 \\
\hline El Salvador & 0.02 & 1.01 \\
Guatemala & 0.61 & 1.20 \\
\hline Honduras & 0.71 & 1.24 \\
\hline Nicaragua & -2.01 & 0.55 \\
\hline Región & 0.28 & 1.09 \\
\hline
\end{tabular}

Fuente: Elaboración propia, WDI 2006.

Siguiendo con la evolución de la distribución del producto per cápita se estimaron las correlaciones de las tasas de crecimiento por periodos decenales entre 1975 y 2005.

Cuadro $\mathrm{N}^{\circ} 2$. Correlación de las tasas de crecimiento

Períodos decenales, 1975-2005

\begin{tabular}{|c|c|c|c|}
\hline & 1975-1985 & $1985-1995$ & $1995-2005$ \\
\hline $1975-1985$ & 1 & & \\
\hline $1985-1995$ & 0.58 & 1 & \\
\hline 1995-2005 & -0.27 & -0.26 & 1 \\
\hline
\end{tabular}

Fuente: Elaboración propia, WDI 2006.

Los resultados son contrastantes, por un lado, existe correlación positiva entre las tasas de crecimiento de 1975-1985 y 1985-1995 y por otro, correlación negativa entre 1985-1995 y 1995-2005. Lo primero se explica porque las tasas de crecimiento crecieron en el período de 1985-1995 y lo segundo se debe a una contracción de las tasas en el último periodo. Reflejo de la volatilidad del crecimiento en la región y a pesar de la negatividad en la correlación se podría interpretar que las desigualdades en la distribución del producto per cápita en la región aumentan, como se verá adelante. 
Otra forma de constatar esta desigualdad es calculando el nivel del PIB per cápita de Costa Rica en relación al resto de países de la región. En el gráfico $\mathrm{N}^{\circ} 3$ se puede distinguir que la tendencia es a la desigualdad regional. Aumenta la brecha entre el nivel del producto per cápita de los países de la región en relación al PIB per cápita de Costa Rica. El Salvador, en 1975, representaba el 80 por ciento del producto per cápita de Costa Rica, treinta años después apenas llega al 50 por ciento. El caso más dramático lo presenta Nicaragua, su PIB per cápita en 1975 estaba por encima al de Costa Rica, en 2005, esta proporción se redujo hasta llegar a un 40 por ciento del producto de Costa Rica.

Gráfico $N^{\circ}$ 3. PIB per cápita de El Salvador, Guatemala, Honduras y Nicaragua relativas a Costa Ri

ca, 1975-2005).

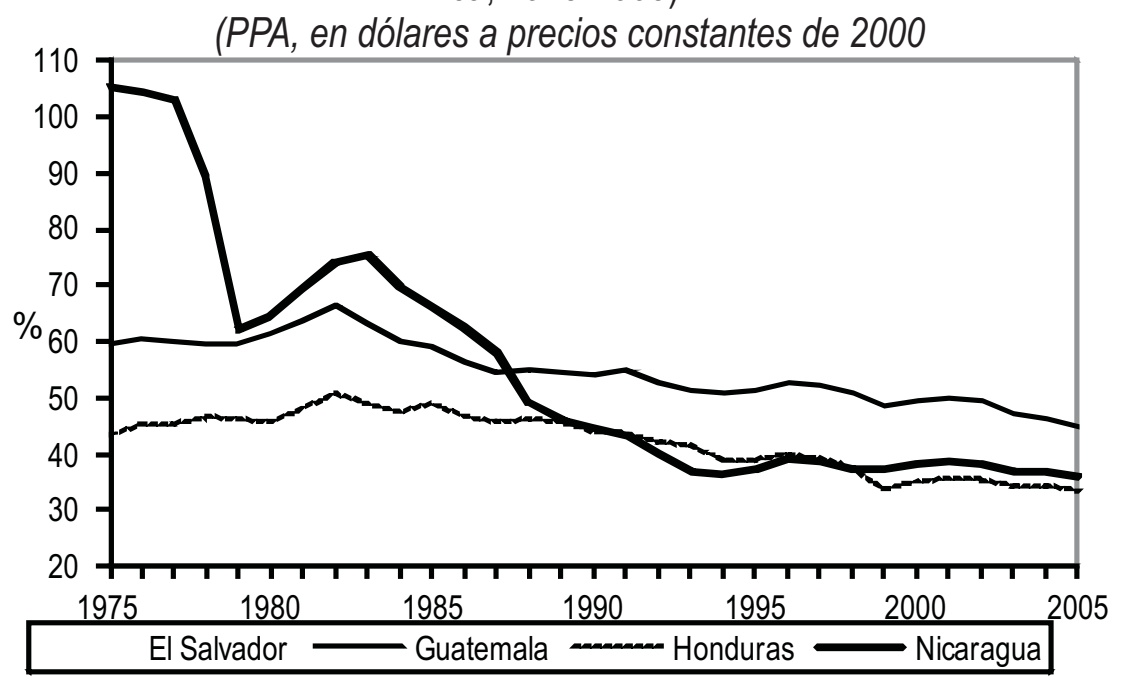

Fuente: Elaboración propia, WDI 2006.

Continuando con el análisis de la evidencia empírica se presentan los resultados de las pruebas de convergencia para los cinco países de la región (Costa Rica, El Salvador, Guatemala, Honduras y Nicaragua). El gráfico $\mathrm{N}^{\circ} 4$ resume los resultados de la regresión de convergencia no condicionada en la que la variable dependiente es la tasa promedio del PIB per cápita durante el periodo de 1975 al 2005. La pendiente negativa de la recta ajustada de la regresión indica que en promedio el ritmo de crecimiento ha sido mayor en los países con un producto per cápita inicialmente menor, este es el caso de Honduras y Guatemala, indicando que presentaron tasas de crecimiento mayor que las del promedio regional. 
Estos países tuvieron un desempeño que produjo convergencia al reducir sus diferencias con el resto. A pesar de esto, es Costa Rica quien tuvo un mejor desempeño que el promedio, ya que era uno de los países con mayor PIB per cápita en 1975 y fue el que mayor crecimiento tuvo en la región, es decir, se hizo más rico en términos relativos. Por su parte, Nicaragua muestra una de las caídas más drásticas del PIB per cápita del total de países de la zona. La hipótesis de convergencia señala que los países con mayores niveles iniciales de PIB per cápita tenderían a crecer a ritmos menores, pero lo que se observa con Nicaragua no es un menor crecimiento, sino una contracción de la actividad económica probablemente marcada por un periodo de crisis. De acuerdo a estudios de la CEPAL (2004), esto se debió probablemente a factores como una hiperinflación durante toda la década de 1980, además de la incertidumbre y la inestabilidad política que influyeron negativamente en su actividad productiva e inversión.

Gráfico № 4. Convergencia Beta del PIB per cápita, 1975-2005

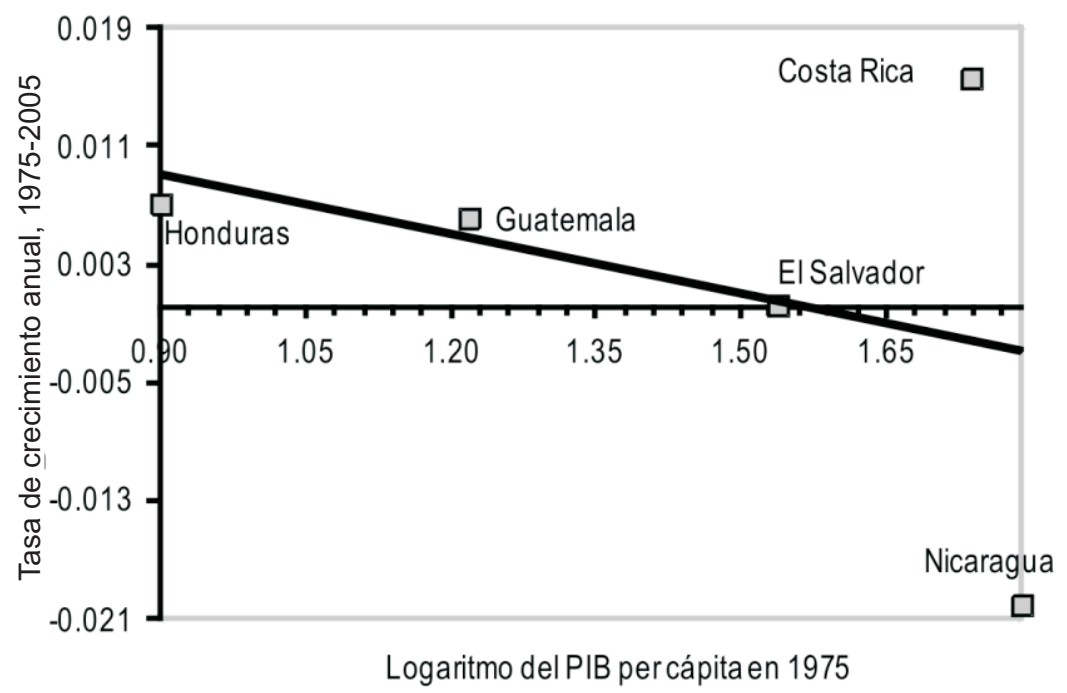

Fuente: Elaboración propia, WDI 2006.

El gráfico $\mathrm{N}^{\circ} 5$ muestra la tendencia en el tiempo de la dispersión del producto per cápita entre la misma muestra de países. La dispersión no es más que la desviación estándar del logaritmo natural del PIB per cápita del período analizado y si la dispersión disminuye con el tiempo se considera evidencia de convergencia $\sigma$ sigma. El patrón de la sigma convergencia muestra una gradual expansión de la desigualdad, sin embargo al final del periodo se aprecia una tendencia hacia la ralentización de la convergencia. 
Gráfico № 5. Convergencia Sigma del PIB per cápita, 1975-2005

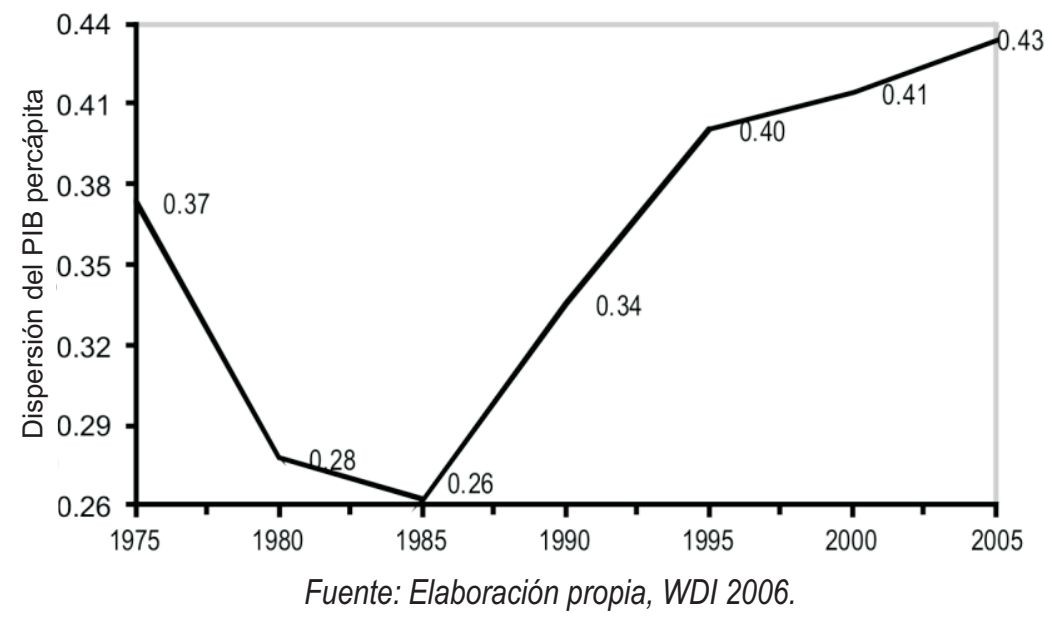

La evolución de la dispersión en el tiempo tiene dos fases claramente diferenciadas: La primera fase que va de 1975 a 1985 se caracterizó por un proceso rápido de convergencia sigma, donde la dispersión decreció de 0,37 hasta el 0,26. En la fase dos, el proceso de convergencia se revierte al pasar la dispersión de 0,26 en 1985 a 0,43 en 2005. El incremento muestra las perturbaciones ocurridas durante la década de 1980 y 1990 en la región. El aumento de la convergencia sigma en este periodo refleja probablemente el efecto de la inestabilidad política sufrida en la zona sobre todo por países como El Salvador, Guatemala y Nicaragua, reduciendo fuertemente su capacidad productiva.

En 1990, la crisis de la deuda, los fenómenos naturales y la caída de los precios agrícolas, como el café afectaron considerablemente las economías de la región, principalmente a Honduras y Nicaragua. En la última década, la dispersión crece, pero tiende a desacelerarse ya que los ritmos de crecimiento son menores que en años anteriores. Los resultados nos indican que el proceso de sigma convergencia regional después de 1980 se ha detenido y a partir de entonces la distribución regional del producto per cápita se desequilibra. En el cuadro $\mathrm{N}^{\circ} 3$ se observan los resultados de las estimaciones de las regresiones no lineales para la misma muestra de países, en periodos sucesivos, desde 1975 hasta el 2005. En cada columna se presentan los valores de los estimadores, sus errores estándar y los $\mathrm{R}^{2}$ de las regresiones. 
Cuadro N³. Resultados de las pruebas de convergencia.

Períodos decenales y quinquenales 1965-2005

\begin{tabular}{|c|c|c|c|}
\hline Períodos & $\boldsymbol{\beta}$ & $\mathbf{R}^{2}$ & e.e regresión \\
\hline $1975-2005$ & $\begin{array}{c}0.017 \\
(0.032)\end{array}$ & 0.141 & 0.014 \\
\hline $1975-1985$ & $\begin{array}{c}0.060 \\
(0.046)\end{array}$ & 0.512 & 0.019 \\
\hline $1985-1995$ & $\begin{array}{c}-0.023 \\
(0.038)\end{array}$ & 0.091 & 0.025 \\
\hline $1995-2005$ & -0.006 & 0.147 & 0.007 \\
\hline
\end{tabular}

Fuente: Elaboración propia, WDI 2006.

Los estimadores obtenidos de $\beta$, en su mayoría, son negativos y algunos son estadísticamente significativos. Para el período 1975-2005, el valor estimado de Beta es positivo, lo que indica que los países con menor PIB per cápita inicial crecieron más rápidamente que otros países con niveles iniciales de PIB mayores. EI ajuste de la regresión es poco significativo, pero el coeficiente de convergencia, es decir, la pendiente de la recta de regresión, sugiere que el proceso de nivelación del PIB per cápita es lento. El valor de este coeficiente es de 0,017 e indica que cada año se elimina tan solo un 1,7 por ciento del diferencial del producto per cápita con respecto al promedio regional. En otras palabras, con esta velocidad de convergencia se necesitarían 41 años para cubrir la mitad de la brecha entre los países con menor nivel de producto per cápita y los de mayor nivel.

En los períodos decenales las pruebas resultaron contrastantes. En el período 19751985 los resultados indican una tendencia a la convergencia alta y significativa, hecho que se contrapone a una disminución de la velocidad de convergencia y a su escasa significancia estadística para el siguiente período. Una posible explicación a la variabilidad de los valores de beta convergencia sería que en la década de 1970 se presenció un proceso de convergencia que posteriormente pudo haber sido contrarrestado por una tendencia a la divergencia retomada a partir de la segunda mitad de la década de 1980. La convergencia durante esta etapa es por las altas tasas observadas por Honduras y Guatemala, superiores incluso a Costa Rica y a la contracción del crecimiento en Nicaragua y El Salvador. Las estimaciones hechas son congruentes con las tendencias observadas en la evolución de la dispersión del producto per cápita en la región. Dado que no se encontraron estudios previos del tema de convergencia en América Central, se compararon los resultados con 
estudios regionales para América Latina. En el caso estudiado por Cabrera (2002) su análisis se centra en la convergencia absoluta entre los estados de México, las tasas de convergencia oscilan entre 0,035 y 0,073, para períodos decenales entre 19701995. En un documento del ILPES (2004) se hace un recuento de varios estudios de convergencia sobre todo en América del Sur. Las estimaciones para Chile entre 19601996 son de 0,009 y 0,020, valores para períodos decenales. En Colombia los coeficientes obtuvieron valores entre 0,008 y 0,057 , también para períodos decenales entre 1950-1989; estos valores fueron calculados sin incluir dummies regionales. Valores próximos a los encontrados en este trabajo que fluctúan entre 0,006 y 0,060.

Para terminar, se estudian algunos factores explicativos del crecimiento económico regional. Como se definió en el apartado teórico para que exista convergencia condicional habría que encontrar una correlación parcial negativa entre crecimiento y nivel de renta o producto per cápita inicial, condicional al estado estacionario. Utilizando regresiones múltiples se puede llegar a esto, es decir introduciendo variables adicionales al modelo que actúen como aproximativa del estado estacionario. El cuadro 4 utiliza los datos del producto real per cápita, donde la variable dependiente es la tasa media anual de crecimiento del PIB per cápita entre 1993 y 2005, y en las demás columnas se incluyen diferentes variables como explicativas de la tasa de crecimiento. La primera columna incluye el logaritmo del PIB per cápita en 1993, y se observa que el coeficiente inicial es negativo, muy similar al obtenido en el cuadro $\mathrm{N}^{\circ} 3$ en el período de 1995-2005, continúa siendo no muy significativa, esto se podría interpretar como evidencia de convergencia condicional.

Una resultante interesante es que en las estimaciones hechas, el coeficiente del producto interior bruto per cápita inicial se vuelve negativo, incluidas las variables explicativas, a decir de Sala-i-Martín (2000), si se acepta que las variables explicativas están relacionadas con los niveles tecnológicos e institucionales que determinan la posición del estado estacionario, entonces podemos interpretar el hecho de que el coeficiente del producto inicial sea negativo como evidencia de convergencia condicional. En la columna 2 el coeficiente del logaritmo del producto inicial es negativo con un valor del 0,017 . Por otra parte, los signos de los coeficientes de las variables educacionales (PRIM 93 y SEC93) son positivos, lo cual concuerda con la importancia que tiene la educación dentro del modelo de crecimiento económico estudiado. Estadísticamente resulta significativa la estimación dado su $\mathrm{R}^{2}$, pero las velocidades de convergencia muestran que la educación secundaria tiene una mayor incidencia en la tasa de crecimiento que lo que tiene la educación primaria. Al mejorar su nivel de educación y de formación cada persona aumenta el stock de capital humano de una nación y de allí contribuye al mejoramiento de la productividad de la economía nacional, es decir, la productividad privada del capital humano tiene un efecto externo positivo, Romer (2006). La tercera columna incluye el gasto en 
ecuación y salud (GES) y una variable educativa (SEC93), esto se hizo con la finalidad de ver el efecto del gasto social en la tasa de crecimiento de los países de la zona. Los signos de los coeficientes son los esperados y el ajuste de la regresión es muy significativo, el mayor dentro del conjunto de las estimaciones hechas, el efecto que la variable del gasto social tiene sobre el crecimiento es del 15 por ciento, al parecer ha colaborado de forma importante, pero no suficiente, al resultado de la convergencia.

En la columna cuatro, se incluyeron la formación bruta de capital fijo como variable sustituta de la inversión y la tasa de crecimiento de la población más la tasa de depreciación. En el modelo Solow-Swan se hacen predicciones de este tipo utilizando la tasa de ahorro y el crecimiento poblacional para alcanzar el equilibrio del estado estacionario. El coeficiente de FC (Formación bruta de capital) es positivo, $0,068(0,115)$, el crecimiento de la población más la tasa de depreciación es negativo, $-0,021(0,022)$, incluidas estas variables el coeficiente del PIB inicial es negativo y similar a los valores encontrados en las otras columnas. Interpretando los resultados, las predicciones del modelo Solow-Swan se cumplen.

Cuadro $\mathrm{N}^{\circ}$ 4. Resultados de las pruebas de convergencia. Determinantes del crecimiento, 1993-2005

\begin{tabular}{|c|c|c|c|c|}
\hline & (1) & (2) & (3) & (4) \\
\hline Constante & $\begin{array}{c}0.002 \\
(0.012)\end{array}$ & $\begin{array}{l}-0.040 \\
(0.071)\end{array}$ & $\begin{array}{c}-0.022 \\
(0.037)\end{array}$ & $\begin{array}{c}0.034 \\
(0.054)\end{array}$ \\
\hline $\log$ PIB (93) & $\begin{array}{l}-0.008 \\
(0.008)\end{array}$ & $\begin{array}{l}-0.017 \\
(0.013)\end{array}$ & $\begin{array}{c}-0.012 \\
(0.017)\end{array}$ & $\begin{array}{c}-0.010 \\
(0.012)\end{array}$ \\
\hline PRIM 93 & & $\begin{array}{c}0.008 \\
(0.044)\end{array}$ & & \\
\hline SEC 93 & & $\begin{array}{c}0.078 \\
(0.088)\end{array}$ & $\begin{array}{c}0.050 \\
(0.082)\end{array}$ & \\
\hline GES & & & $\begin{array}{c}0.149 \\
(0.394)\end{array}$ & \\
\hline FC & & & & $\begin{array}{c}0.068 \\
(0.115)\end{array}$ \\
\hline$n+\delta$ & & & & $\begin{array}{l}-0.021 \\
(0.022)\end{array}$ \\
\hline $\mathrm{R}^{2}$ & 0.214 & 0.603 & 0.640 & 0.605 \\
\hline E.S regresión & 0.007 & 0.009 & 0.008 & 0.008 \\
\hline
\end{tabular}




\section{Nota:}

Las regresiones corresponden a estimaciones hechas por mínimos cuadrados no lineales de ecuaciones de la siguiente forma:

$$
\left(\frac{\ln y_{i, t}-\ln y_{i, 0}}{T}\right)=\alpha-\left(\frac{1-\ell^{-\beta \cdot T}}{T}\right) * \ln y_{i, 0}+\delta X_{i, 0}
$$

donde $\mathrm{Y}_{\mathrm{i}, \mathrm{t}}$ es el PIB per cápita del país i en el periodo $\mathrm{t}, \mathrm{X}_{\mathrm{i}, 0}$ son variables explicativas que intervienen en la tasa de crecimiento y $T$ es la duración del periodo. Entre paréntesis se presenta el error estándar de cada coeficiente. Las variables incluidas en las estimaciones son las siguientes:

Log PIB 93: logaritmo del PIB per cápita de 1993.

PRIM 93: fracción de la población en edad escolar matriculada en primaria en el año de 1993.

SEC 93: fracción de la población en edad escolar matriculada en secundaria en el año de 1993.

GES: gasto público en salud y educación promedio entre los años 1993 y 2005.

FC: gasto en formación bruta en capital fijo como porcentaje del PIB entre los años 1993 y 2005.

$\mathrm{n}+\boldsymbol{\delta}$ : tasa promedio (1993-2005) del crecimiento poblacional más tasa de depreciación, la cual se ha supuesto constante e igual a 0.05

\section{CONCLUSIONES}

El comportamiento del crecimiento es muy dispar en cada uno de los países analizados, a pesar de haber experimentado tasas de crecimiento positivos, el poder de predicción de la experiencia de crecimiento pasado resulta ser un predictor débil del crecimiento futuro. Al parecer, la inestabilidad del crecimiento económico estaría influyendo en el aumento de las desigualdades en la distribución del producto per cápita en la región.

Por otra parte, en las pruebas de las distintas hipótesis de convergencia para el PIB per cápita los resultados son contrastantes. Así, en el largo plazo existe convergencia en términos absolutos, siendo las estimaciones del coeficiente (beta) en los períodos decenales significativamente diferentes entre sí y las velocidades de 
convergencia decrecen sobre todo en el último período. De acuerdo a Barro y Salai-Martín (1995) lo anterior estaría explicado, por un lado, por los ritmos de crecimiento económicos, los cuales son muy inestables por lo que cualquier cambio produce efectos excepcionales en sus resultados, ya sean negativos 0 positivos. Por otro lado, los llamados cambios exógenos producen fuertes perturbaciones en los procesos de convergencia. En cuanto a los efectos de los determinantes del crecimiento económico, las predicciones se cumplieron. El gasto en educación y salud resultó ser la variable con mayor nivel de influencia dentro de la contrastación de hipótesis de convergencia condicional. Las pruebas muestran una asociación estadística positiva entre ésta y las variables educativas, además que la estimación tiene la mayor significancia dentro del resto de pruebas realizadas. También se constató que la formación bruta de capital está positivamente correlacionada con la tasa de crecimiento.

A raíz de los resultados obtenidos, habría que resaltar que la evolución del crecimiento de los diferentes países analizados es desigual, por lo tanto es difícil hablar de la existencia de un patrón común. La hipótesis de convergencia para el período 1975-2005 resulta ser muy débil y lo contrario sucede en las pruebas posteriores, es decir, divergencia.

\section{BIBLIOGRAFÍA}

Banco Mundial, "Base de datos de los Indicadores de Desarrollo Mundial", 2006.

Barro. R, "Economic Growth in a Cross Section of Countries", NBER Working Papers Series, Working Paper 3120, National Bureau of Economic Research, September 1989.

Barro. R y Sala-i-Martin. X, "Economic Growth”, McGraw-Hill Editors, 1995.

Castellanos Cabrera. L, "Crecimiento Económico y Convergencia Regional en México" (1970-1995), en Anuario de la DCSEA 2001. ISBN: 9686148582. Editorial TallerAbierto, México 2002.

Díaz. R y Meller. P, "Crecimiento Económico Regional en Chile: ¿Convergencia?", Centro de Economía Aplicada, Universidad de Chile, Documentos de Trabajo No.180, enero 2003.

Esquivel. G, "Convergencia Regional en México, 1940-1995", El Trimestre Económico, Vol. 66, No 264, octubre-diciembre, México 1999.

Mankiw. G, Romer. D y Weil. D, "A Contribution to the Empirics of Economic Growth", NBER Working Papers Series, Working Paper 3541, National Bureau of Economic Research, December 1990.

Sala-i-Martín, X. "Apuntes de Crecimiento Económico", Antoni Bosch Editor $2^{\mathrm{a}}$ Edición, Barcelona 2000. 